\title{
Evidence for a Morphological Evolution of Spiral Galaxies
}

\author{
Yvan Dutil \\ Departamento de Matematica Aplicada II Facultad de Matematicas y \\ Estadistica - Edificio U Pau Gargallo 5, E-08028 Barcelona, Spain \\ Jean-René Roy \\ Dept. de Physique, Université Laval, Quebec, PQ G1K 7P4, Canada
}

\begin{abstract}
A detailed analysis of the oxygen abundance profile has been carried out on a sample of spiral galaxies from which very good data was available. The early-type galaxies of our sample display gradients that are flatter, and overall levels of $\mathrm{O} / \mathrm{H}$ abundances that are higher, than those of normal late-type galaxies. Early-type galaxies show an identical trend in the behavior of extrapolated central abundance versus morphological type to that shown by late-type galaxies with strong bars, even in the absence of a bar. On a diagram showing extrapolated central abundances versus morphological types, two clearly separated sequences appear: latetype barred galaxies and early-type (barred or unbarred) galaxies clearly fall on a sequence 0.5 dex in abundance below that of normal late-type galaxies. This behavior is consistent with theoretical models of morphological evolution of disk galaxies by the formation and dissolution of a bar over a period of a few $10^{9} \mathrm{yr}$, where later type galaxies (Sd, Sc, SBc) evolve into earlier-type disk galaxies $(\mathrm{Sb}, \mathrm{Sa})$ through transitory $\mathrm{SBc}$ and $\mathrm{SBb}$ phases.
\end{abstract}

\section{Discussion}

Charley Lineweaver: This morning, Cowie told us that as the column density of neutral hydrogen goes down so does the metallicity. Here you are showing a decrease in metallicity with galactic radius. Can you say anything about the relation of these two?

Yvan Dutil: It is not clear. I would have to do a calculation based on the variation of the $\mathrm{HI}$ column density with radius. However, low-mass galaxies have lower abundances. This may partly answer the question. 